\title{
Clinical impact of endemic NDM-producing Klebsiella pneumoniae in intensive care units of the national referral hospital in Jakarta, Indonesia
}

Yulia Rosa Saharman ${ }^{1,2}$, Anis Karuniawati ${ }^{1}$, Rudyanto Sedono ${ }^{3}$, Dita Aditianingsih ${ }^{3}$, Wil H. F. Goessens ${ }^{2}$, Corné H. W. Klaassen², Henri A. Verbrugh ${ }^{2}$ and Juliëtte A. Severin ${ }^{2 *}$

\begin{abstract}
Objective: A prospective observational study was performed to assess the epidemiology and clinical impact of carbapenem-non-susceptible Klebsiella pneumoniae (CNKP) in intensive care units (ICUs) of the national referral hospital in Jakarta, Indonesia.
\end{abstract}

Materials/methods: Adult patients consecutively hospitalized for $>48 \mathrm{~h}$ in two ICUs of the national referral hospital were included from April until October 2013 and from April until August 2014. K. pneumoniae from clinical cultures and standardized screening of rectum and throat on admission, discharge and weekly if hospitalized $>7$ days were collected. Environmental niches and healthcare workers (HCWs) were also screened. Susceptibility was determined phenotypically and the presence of carbapenemase genes by PCR. Raman spectroscopy as well as multiple-locus variable number tandem repeat analysis (MLVA) were used for typing.

Results: Twenty-two out of 412 (5.3\%) patients carried CNKP on admission and 37/390 (9.5\%) acquired CNKP during ICU stay. The acquisition rate was 24.7/1000 patient-days at risk. One out of 31 (3.2\%) environmental isolates was a CNKP. None of the HCWs carried CNKP. Acquisition of CNKP was associated with longer ICU stay (adjusted Hazard Ratio: 2.32 [Clgg: 1.35-3.68]). ICU survival was lower among patients with CNKP compared to patients with carbapenem-susceptible K. pneumoniae (aHR 2.57, $p=0.005$ ). Ninety-six of the 100 (96\%) CNKP isolates carried a carbapenemase gene, predominantly b/a $a_{\mathrm{NDM}}$. Raman typing revealed three major clusters among 48 Raman types identified, whereas MLVA distinguished six major clusters among a total of 30 different genotypes.

Conclusions: NDM-producing CNKP are introduced into these ICUs and some strains expand clonally among patients and the environment, resulting in endemic CNKP. CNKP acquisition was associated with prolonged ICU stay and may affect ICU survival.

(Continued on next page)

\footnotetext{
* Correspondence: j.severin@erasmusmc.nl

Preliminary results of the study were presented at the $26^{\text {th }}$ ECCMID 2016 in

Amsterdam, The Netherlands (EV0366).

2Department of Medical Microbiology and Infectious Diseases, Erasmus MC

University Medical Center Rotterdam, Dr. Molewaterplein 40, 3015, GD,

Rotterdam, The Netherlands

Full list of author information is available at the end of the article
}

(c) The Author(s). 2020 Open Access This article is licensed under a Creative Commons Attribution 4.0 International License, which permits use, sharing, adaptation, distribution and reproduction in any medium or format, as long as you give appropriate credit to the original author(s) and the source, provide a link to the Creative Commons licence, and indicate if changes were made. The images or other third party material in this article are included in the article's Creative Commons. licence, unless indicated otherwise in a credit line to the material. If material is not included in the article's Creative Commons licence and your intended use is not permitted by statutory regulation or exceeds the permitted use, you will need to obtain permission directly from the copyright holder. To view a copy of this licence, visit http://creativecommons.org/licenses/by/4.0/ The Creative Commons Public Domain Dedication waiver (http://creativecommons.org/publicdomain/zero/1.0/) applies to the data made available in this article, unless otherwise stated in a credit line to the data. 
(Continued from previous page)

Trial registration: The study was registered at Netherlands Trial Register http://www.trialregister.nl. Candidate number: 23527, NTR number: NTR5541, NL number: NL5425 (https://www.trialregister.nl/trial/5424), Retrospectively registered: NTR: 22 December 2015.

Keywords: Klebsiella pneumoniae, Microbial drug resistance, Carbapenemase, Intensive care unit, Mortality, Indonesia

\section{Introduction}

Carbapenems are the antibiotics of choice for treatment of life-threatening infections due to multidrug-resistant Gram-negative bacilli. However, the worldwide emergence of carbapenem-non-susceptible Klebsiella pneumoniae, especially in intensive care units (ICUs), has become a major challenge. Non-susceptibility to carbapenems in $K$. pneumoniae may be due to production of Ambler class A $\beta$-lactamases (e.g. KPC), class B metallo$\beta$-lactamases (MBLs, e.g. VIM, IMP, NDM) or class D oxacillinases (e.g. OXA-48 like enzymes) [1-3].

Although carbapenemase-producing $K$. pneumoniae have emerged globally, geographic variations do exist. K. pneumoniae producing KPCs have initially mainly been reported in the USA and Israel, but more recently also from China and Taiwan [1, 2, 4, 5]. $K$. pneumoniae strains carrying OXA-48-like carbapenemases were first described in Turkey in 2003. Currently, K. pneumoniae with OXA-48-like carbapene mases are spreading rapidly in many European countries, in addition to being endemic in the Middle East and in Northern Africa [1, 4, 5]. Bacteria with the New Delhi metallo- $\beta$-lactamase (NDM) enzyme, which was first identified in Sweden from a patient who had travelled from New Delhi, India, have attained endemic levels in countries of the Indian subcontinent including India, Pakistan, Bangladesh and Sri Lanka $[1,4-8]$. This gene is also encountered in bacteria, including $K$. pneumoniae, in some countries in the South East Asian region, including Singapore [9], Thailand [10] and Vietnam [1]. However, so far there have been few data on the epidemiology of carbapenem-non-susceptible $K$. pneumoniae reported from Indonesia, the fourth most populous country in the world. In 2011, 27.6\% of the Enterobacteriaceae isolated from specimens at two ICUs in Jakarta was carbapenem-resistant, including one $K$. pneumoniae harboring the $b l a_{\mathrm{NDM}}$ gene [11]. In 2014-2015, the prevalence of resistance to meropenem among $K$. pneumoniae from urinary tract infections in clinical and outpatient clinical settings was $14.0 \%$, but no further analysis of these isolates was performed [12]. The aim of the present study was to delineate the clinical and molecular epidemiology of carbapenemnon-susceptible $K$. pneumoniae isolated in two ICUs of the Dr. Cipto Mangunkusumo General Hospital, the national referral teaching hospital in Jakarta.

\section{Materials and methods Study design}

A prospective observational study was performed in a 1000-bed national referral teaching hospital with 34,000 admissions per year in Jakarta, Indonesia, from April until October 2013 and from April until August 2014. Two ICUs participated, the adult ICU and the Emergency Room (ER)-ICU, with 865 and 390 admissions in 2013, respectively, and 1154 and 439 admissions in 2014, respectively. The adult ICU is a 12-bed open ward with mechanical ventilation facilities, admitting patients with various medical and surgical indications, and one designated nurse per patient during morning shifts and a 1:1.5 nurse/patient ratio during other shifts. It is also used as post-anaesthetic care unit. The ER-ICU has the same design, but 8 beds, and the nurse per patient ratio in the morning shifts is $1: 1$ and during the other shifts $1: 2$. This ICU is also used for short observations.

All adult patients ( $\geq 18$ years old) admitted to one of the two ICUs and hospitalized for more than $48 \mathrm{~h}$ were eligible for enrollment in this study. Informed consent was obtained from the patient or their relatives as applicable. Demographic and clinical characteristics such as age, gender, medical or surgical indication, underlying diseases, hospitalization history, and previous use of antibiotics on admission were recorded.

Systemic inflammatory response syndrome (SIRS) criteria on admission were used as a screening tool to assess (severity of) septic illness. The "Acute Physiology and Chronic Health Evaluation II" score was not feasible in this low-resource setting. SIRS is defined as two or more of the following: fever $>38^{\circ} \mathrm{C}$ or $<36^{\circ} \mathrm{C}$, heart rate $>90$ beats per minute, respiratory rate $>20$ breaths per minute or $\mathrm{PaCO} 2<32 \mathrm{mmHg}$, abnormal white blood cell count ( $>12,000 / \mathrm{mm}^{3}$ or $<4000 / \mathrm{mm}^{3}$ or $>10 \%$ bands) [13].

The quick Sequential Organ Failure Assessment (qSOFA) score is a newer bedside prompt that may identify patients with suspected infection and helps to determine sepsis in all healthcare environments. The qSOFA score assigns one point for each of the 
following conditions: systolic blood pressure $\leq 100$ $\mathrm{mmHg}$, respiratory rate $\geq 22$ breaths per minute, and altered mentation (Glasgow coma scale <15). The score ranges from 0 to 3 points. A qSOFA score $\geq 2$ at the onset of infection is associated with a greater risk of death and prolonged ICU stay. This score was included as well [13].

Acquisition is defined as a screening culture (throat or rectum/stool) or clinical culture with a first detection of $K$. pneumoniae with reduced susceptibility to a carbapenem, that was not present in cultures taken from the patient on admission or in the first $48 \mathrm{~h}$ of admission. Outcome measures were acquisition of a carbapenem-susceptible and carbapenem-non-susc eptible K. pneumoniae (independent of resistance to other classes of antibiotics), length of stay in the ICU, and mortality during ICU stay.

Environmental samples were taken twice (in October 2013 and December 2014), simultaneously in both ICUs. Screening of healthcare workers (HCWs) was performed once. HCWs were defined as all personnel including doctors, nurses and other people (cleaning staff, administration staff, porters, nutritionist) working in one of the two ICUs during the study period.

\section{Sampling}

From patients enrolled, screening cultures were obtained from throat and rectum or stools by experienced ICU nurses on the day of admission, at the time of discharge from the ICU, and weekly if the patient was admitted for seven days or more. The samples were collected with sterile cotton-tipped swabs and placed in Amies transport medium (Oxoid, Basingstoke, UK). The swabs were transported in clean, closed boxes at ambient temperature to the laboratory on the same day. All swabs were processed in the laboratory within $24 \mathrm{~h}$.

Clinical samples were collected from a patient when the ICU physician suspected the patient of having an infection. Specimens were taken under aseptic precautions from the lower respiratory tract, blood, urine, tissue, or wound, on indication.

Environmental samples were taken from various sites, including wash basins, bed rails, bedside cabinet tables, ventilators, and monitor screens (Supplementary Table 1), with sterile cotton-tipped swabs and placed in Amies transport medium [14].

All HCWs working in one of the ICUs were sampled (rectal and throat) once over the course of one month (September 2013) with sterile cotton-tipped swabs, which were transported to the laboratory in Amies transport medium.

\section{Microbiological methods}

\section{Isolation and identification of bacteria}

In the laboratory, each screening swab was placed in a trypticase soy broth (TSB) supplemented with cefotaxime $2 \mathrm{mg} / \mathrm{L}$ plus vancomycin $50 \mathrm{mg} / \mathrm{L}$ and incubated overnight. The next day, a loop of broth was subcultured on MacConkey agar (Oxoid).

Blood cultures were collected in BACTEC (BD, Franklin Lakes, NJ, USA) bottles as per manufacturer's instructions with a minimum of $10 \mathrm{~mL}$ of blood collected from at least two puncture sites. Other clinical specimens were inoculated onto blood and MacConkey agar plates (Oxoid) and incubated for $24 \mathrm{~h}$ at $37^{\circ} \mathrm{C}$. All morphologically different colonies were examined by Gram stain and identified using the VITEK2 ${ }^{\circ}$ system (bioMérieux, Lyon, France).

Strains were stored in duplicate in $-80^{\circ} \mathrm{C}$ in TSB with glycerol $10 \%$. One tube of each strain was sent to the Department of Medical Microbiology and Infectious Diseases, Erasmus MC, Rotterdam, the Netherlands, for further analysis. The other tube of each strain remained in the Indonesian laboratory. In the Netherlands, the identity of strains was confirmed using matrix-assisted laser desorption/ionisation (Maldi Biotyper, Bruker Microflex LT, Bruker, London, UK).

The quality control strains used for this part of the study in Indonesia were Escherichia coli ATCC 25922 and Pseudomonas aeruginosa ATCC 27853, in the laboratory of Erasmus MC multiple quality control strains were used.

\section{Antimicrobial susceptibility testing}

Imipenem and meropenem susceptibility tests on isolates from screening cultures were performed by standard KirbyBauer disc diffusion technique using Mueller-Hinton agar plates (BD) based on EUCAST Disc Diffusion Method for Antimicrobial Susceptibility Testing- Version 3.0 (April 2013). Minimum inhibitory concentrations (MICs) of antibiotics were determined by VITEK2 ${ }^{\circ}$ for clinical isolates. Carbapenem MICs and zone sizes were interpreted according to EUCAST (2013) using the following breakpoints for non-susceptibility: meropenem $>0.25 \mathrm{mg} / \mathrm{L}(<24 \mathrm{~mm})$, imipenem $>1 \mathrm{mg} / \mathrm{L}(<22 \mathrm{~mm})$ [15].

For this part of the study, quality control strains as described above were used.

\section{String test}

In order to determine hyper-muco-viscosity, the string assay was performed for all $K$. pneumoniae isolates. For this, the strains were inoculated onto $5 \%$ sheep blood agar (BD) and kept overnight at $37^{\circ} \mathrm{C}$. An individual colony was then touched with a $1 \mu \mathrm{L}$ disposable loop which was subsequently pulled up slowly. The string test was 
deemed positive if a string of $\geq 5 \mathrm{~mm}$ was formed between the colony and the loop [16].

\section{Phenotypic detection of carbapenemase}

A phenotypic detection test for Ambler class A and B and OXA-48-like carbapenemases was performed with discs (Rosco Diagnostica A/S, Taastrup, Denmark) containing meropenem $(10 \mu \mathrm{g})$, temocillin $(30 \mu \mathrm{g})$, meropenem + phenyl boronic acid (PBA), meropenem + dipicolinic acid (DPA), meropenem + PBA + DPA, and meropenem + cloxacillin (CL), using a 0.5 McFarland suspension of the isolates on Mueller Hinton II agar plates. Zone diameters were measured after overnight incubation at $37^{\circ} \mathrm{C}$. The temocillin zone diameter was only interpreted if no synergy was observed with DPA and/or PBA. Isolates without synergy with the PBA or DPA test and a temocillin zone diameter $\leq 10 \mathrm{~mm}$ (i.e. the absence of an inhibition zone around the temocillin disc) were considered OXA carbapenemase positive. The interpretation of the PBA and DPA synergy tests and the temocillin disc diffusion were as described previously [17].

\section{DNA extraction and PCR for carbapenemase genes}

DNA from the isolates was extracted by a cell lysis step and boiling using the InstaGene Matrix (Bio-Rad Laboratories, USA) according to the manufacturer's instructions. PCR-based detection of Ambler class A carbapenemases $\left(b l a_{\mathrm{KPC}}\right)$, Ambler class $\mathrm{B}$ metallo- $\beta$ lactamases $\left(b l a_{\mathrm{NDM}}\right)$, and class $\mathrm{D} \quad \beta$-lactamases (bla $\left.a_{\text {OXA-48-like }}\right)$ were carried out using T3000 Thermocycler (Biometra-Whatman, Goettingen). PCR primers and reaction conditions for PCR were as described previously [18-20]. Amplified PCR products were resolved by electrophoresis at $250 \mathrm{~V}$ for $30 \mathrm{~min}$ on $1.5 \%$ agarose gels with $0.5 \mathrm{x}$ Tris $(89 \mathrm{mM})$-boric acid $(89 \mathrm{mM})$-EDTA (2 $\mathrm{mM}$ ) buffer containing $\mathrm{SyBr}^{\circledR}$ Safe DNA Gel Stain and visualized under UV light and photographed. In each run, a positive and negative control was included.

\section{Clonal relatedness}

Raman spectroscopy (SpectraCell RA ${ }^{\circ}$ Bacterial Strain Analyzer, RiverD International BV, Rotterdam, The Netherlands) was applied as a first typing method [21, 22]. All isolates were grown overnight on trypticase soy agar (TSA; BD). Samples were prepared and submitted to spectrometry as described previously [22]. Raman light scatterings were analyzed by SpectraCellRA software version 1.9.0.13444: 24 (RiverD). The similarity between pairs of spectra was calculated using the squared Pearson correlation coefficient $\left(\mathrm{R}^{2}\right.$-values), multiplied by 100 and expressed as a percentage. The similarity threshold for this study was set at $91 \%$ so that two isolates with an $\mathrm{R}^{2}$ below this threshold were considered to be different and were designated different Raman types. Two isolates with an $\mathrm{R}^{2}$-value above $99.5 \%$ were considered indistinguishable and were considered to have the same Raman type. In case of an $R^{2}$ value between of 91 and $99.5 \%$, these isolates were considered highly related but not identical [23].

Correlation matrices displayed as 2D plots diagram were created using MATLAB version 7.1 (The MathWorks, Natick, MA, USA).

Multiple-locus variable number tandem repeat analysis (MLVA) was used as a second typing method. The MLVA typing protocol was based on Brink et al. [24] with minor modifications (for details, see Supplement). DNA was quantitated using PicoGreen dsDNA reagent (Invitrogen, Bleiswijk, The Netherlands). Amplification reactions contained approximately $1 \mathrm{ng}$ of DNA and primers according to Supplementary Table 2 in $1 \mathrm{x}$ Roche FastStart PCR Master Mix (Roche diagnostics, Almere, The Netherlands). The thermocycling protocol consisted of an initial denaturation for $5 \mathrm{~min}$ at $95^{\circ} \mathrm{C}$ followed by 30 amplification cycles of denaturation for $30 \mathrm{~s}$ at $95^{\circ} \mathrm{C}$, 30s annealing at $58^{\circ} \mathrm{C}$ and 1 min extension at $72{ }^{\circ} \mathrm{C}$. A final extension step of $30 \mathrm{~min}$ at $72^{\circ} \mathrm{C}$ was applied before reactions were cooled to room temperature. Before loading, amplification products were diluted 100x, combined with the GeneScan 600 LIZ Dye Size Standard (ThermoFisher Scientific, Bleiswijk, The Netherlands) and run on an ABI 3130 capillary electrophoresis platform (ThermoFisher Scientific) using recommended conditions. Electropherograms were analyzed using the MLVA plugin in BioNumerics v7.6 software (Applied-Maths, Sint-Martens-Latem, Belgium). Assignment of repeat numbers was calibrated by comparing our results to those obtained with selected isolates that were genotyped by the Maastricht lab. Typing data was analyzed categorically.

\section{Statistical analysis}

Statistical analyses were performed using SPSS Version 24.0 (SPSS, Chicago, IL, USA). Baseline characteristics from patients admitted to the adult ICU were compared to those in the ER-ICU using Chi square and MannWhitney as appropriate. One-way ANOVA was used to compare patient characteristics according to their $K$. pneumoniae status. Univariate and multivariate analyses were performed to establish risk factors associated with in-ICU mortality using a multivariate logistic regression model with backward selection and inclusion of variables with a $p$ value $<0.1$ in the univariate analysis. Cox proportional regression was used to analyze risk factors for length of stay. Kaplan-Meier method was performed to construct survival curves. The R-code (R-3.6.2.pkg. binary for OS X 10.11 software can be obtained via CRAN, the Comprehensive $\mathrm{R}$ Archive Network, http://cran.R- 
project.org) was used to calculate the competing risks estimates (competing risk analysis is available in an addon package called cmprsk) of the cumulative incidence function and conditional probability function for ICU discharge and in-ICU mortality $[25,26] . P$ values less than 0.01 were considered significant [27].

\section{Results}

\section{Patient characteristics and outcomes}

During the 11-month study period, 1211 patients were hospitalized in the ICUs (Adult ICU: 863, ER-ICU: 348). Of the 412 included patients, 188 were admitted to the adult ICU and 224 to the ER-ICU. Supplementary Table 3 shows baseline characteristics of included patients in each ICU. There were no significant differences in characteristics between patients in both ICUs, except that in the adult ICU most of the patients had been referred from another ward in the same hospital and the proportion of patients with malignancies was higher. Therefore, we analyzed the data from the ICUs both separately and pooled.

Overall, 192/412 (46.6\%) patients had at least one positive culture with $K$. pneumoniae, the remaining 220 patients were free from $K$. pneumoniae on admission and remained so during their ICU stay. One hundred (24.3\%) patients already carried $K$. pneumoniae on the day of admission, of whom 78 carried a carbapenem-susceptible
K. pneumoniae and 22 (5.3\%) carried a carbapenemnon-susceptible $K$. pneumoniae strain (Supplementary Figure 1). One hundred patients $(32.1 \%)$ acquired $K$. pneumoniae during ICU stay, a carbapenem-nonsusceptible $K$. pneumoniae strain in 37 cases and a carbapenem-susceptible strain of $K$. pneumoniae by 63 patients. Thus, a total of 59 patients (14.3\%) carried a carbapenem-non-susceptible $K$. pneumoniae at a certain moment during their ICU stay. In 44 patients, this $K$. pneumoniae was only found in a screening culture, in five patients only from a clinical specimen, and in ten patients from both screening and clinical samples.

The dynamics of acquisition of $K$. pneumoniae in the ICUs is shown in Fig. 1. Patients that acquired a carbapenem-susceptible $K$. pneumoniae had their first positive culture approximately four days sooner than patients that acquired a carbapenem-non-susceptible strain of $K$. pneumoniae $(p<0.001)$. However, the acquisition rate of carbapenem-susceptible $K$. pneumoniae was higher with 41.0 per 1000 patient-days at risk (adult ICU: 46.1; ER-ICU: 35.9) compared to the acquisition rate of carbapenem-non-susceptible $K$. pneumoniae that was 24.7 per 1000 patient-days at risk (adult ICU: 22.2; ER-ICU: 27.0).

Patient outcomes were clearly associated with $K$. pneumoniae status. Patients who acquired carbapenem-nonsusceptible $K$. pneumoniae during ICU stay had a

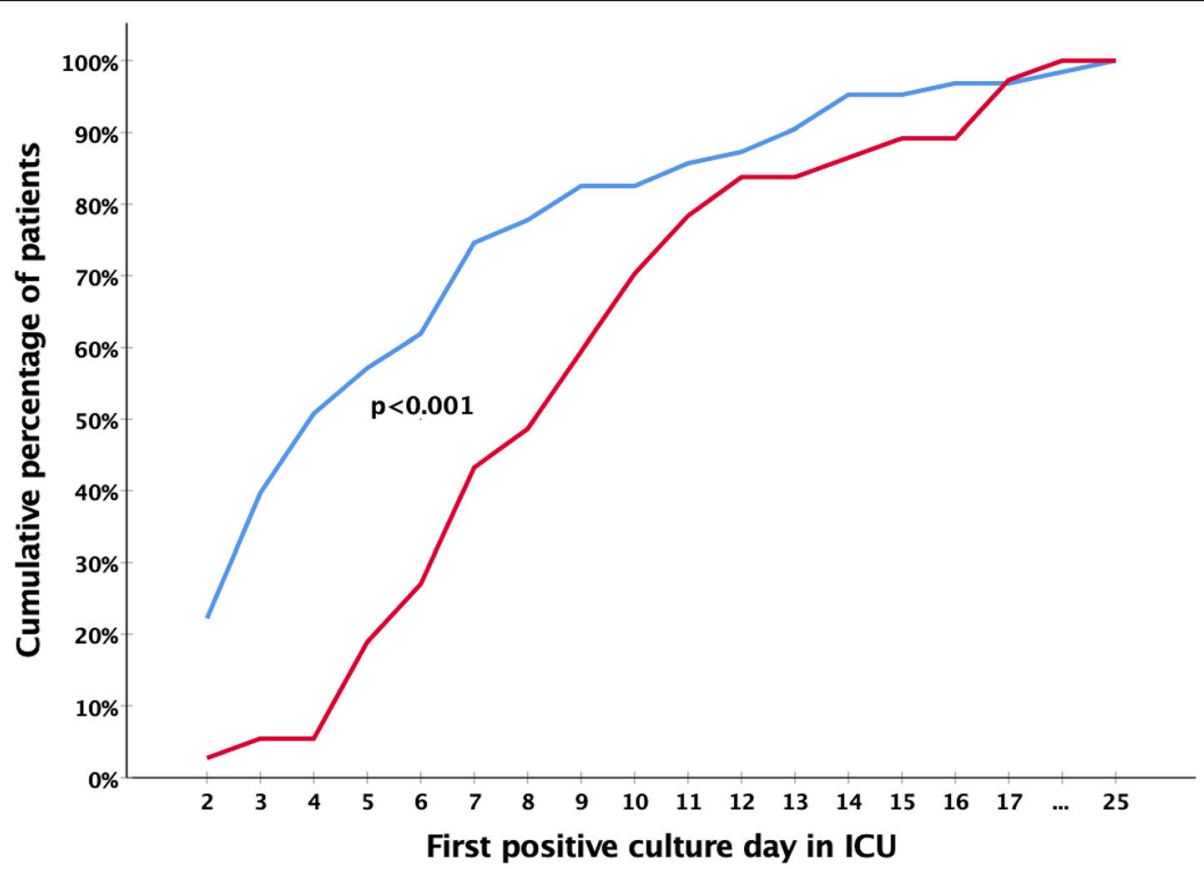

Fig. 1 Rate of acquisition of carbapenem-susceptible and -non-susceptible K. pneumoniae in ICUs. Acquisition dynamics of carbapenemsusceptible and -non-susceptible K. pneumoniae during ICU stay. The blue line represents the cumulative percentage of patients by first day of culture being positive for carbapenem-susceptible K. pneumoniae during ICU stay. The red line represents the cumulative percentage of patients by first day of culture being positive for carbapenem-non-susceptible $K$. pneumoniae during ICU stay. $P$ value was calculated using independent samples-Mann Whitney $U$ test. In total, data from 100 patients are included in this figure 


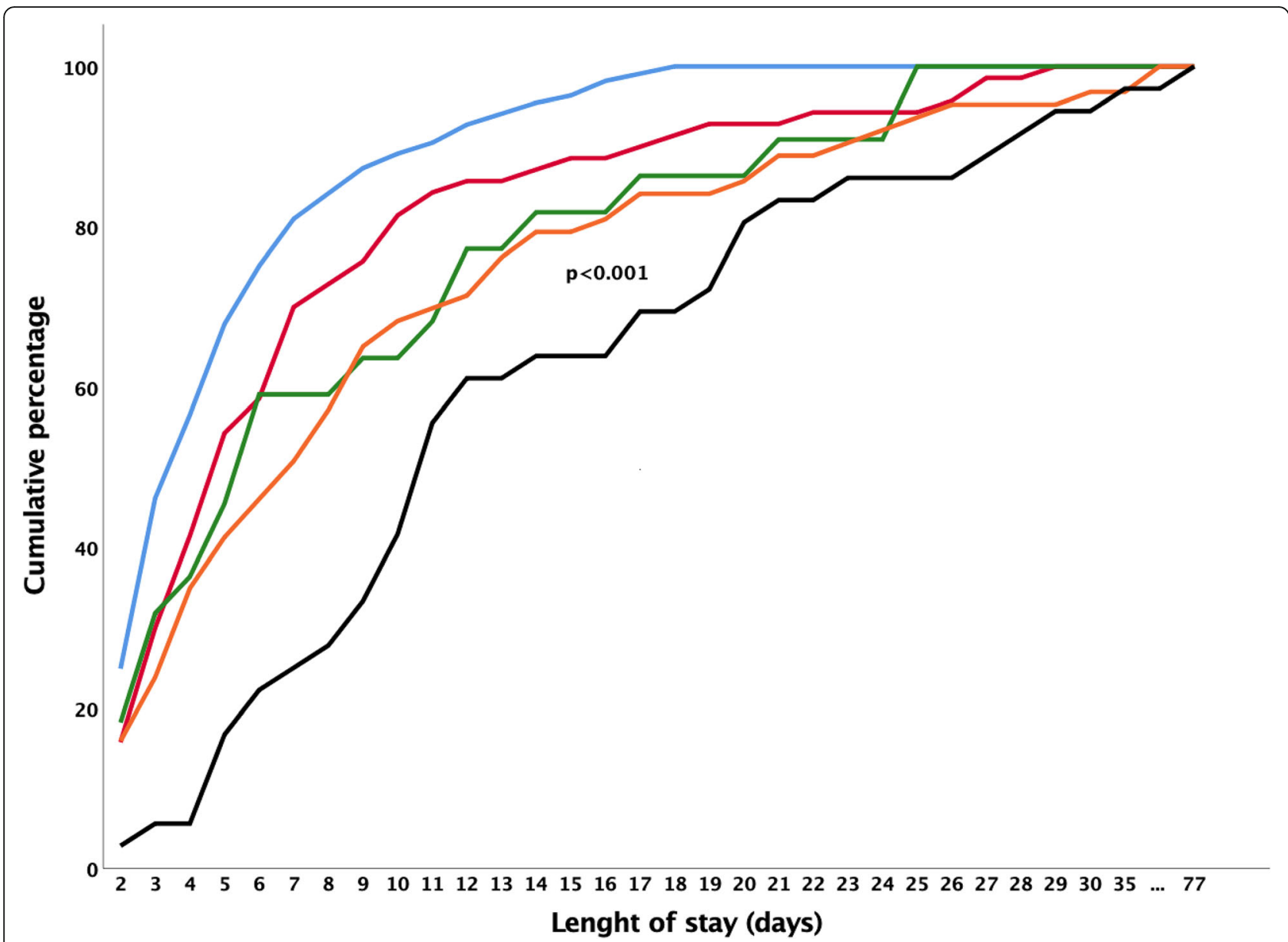

Fig. 2 Cumulative percentage of length of stay according to K. pneumoniae status. Cumulative length of ICU stay of patients based on their $K$. pneumoniae status. Length of stay (days) represent total days patients were hospitalized in the ICU. The blue line represents patients that were always K. pneumoniae negative during their ICU stay. The red line represents patients already positive for carbapenem-susceptible K. pneumoniae on the day of admission. The green line represents patients already positive for carbapenem-non-susceptible $K$. pneumoniae on the day of admission. The orange line represents patients that acquired carbapenem-susceptible K. pneumoniae during ICU stay and the black line represents patients that acquired carbapenem-non-susceptible $K$. pneumoniae during ICU stay. The length of stay of patients that became positive with carbapenem-non-susceptible $K$. pneumoniae during ICU stay was longer than that of the other groups (Cox regression, $P<0.001$ )

significantly longer length of stay (median [interquartile range (IQR)]: 11 [8-20] days, adjusted Hazard Ratio [aHR]: 2.32 [99\% confidence interval (CI): 1.35-3.68], $p<0.001$, Fig. 2 and Supplementary Table 4) compared to the other groups of patients, of whom $\geq 80 \%$ were discharged from the ICU within 2-13 days. Interestingly, these latter groups included the patients that were always free from $K$. pneumoniae, and patients that already carried K. pneumoniae (either carbapenem-susceptible or carbapenem-non-susceptible) at the time of admission to the ICU and patients who became positive for carbapenem-susceptible $K$. pneumoniae during their stay in ICU (Fig. 2).

A longer length of stay was also associated with mechanical ventilation $\geq 5$ days (median [IQR]: 10 [7-15],
aHR: 2.79 [ $\left.\mathrm{Cl}_{99}\right]$ : 1.80-4.34], $p<0.001$, Supplementary Table 4 ) and use of a urinary catheter $\geq 5$ days (median

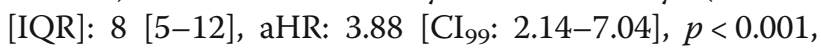
Supplementary Table 4) during ICU stay.

However, the acquisition of $K$. pneumoniae was not associated with in-ICU mortality, $30.5 \%$ of patients that remained free of $K$. pneumoniae died versus 17.5 and $43.2 \%$ of patients that acquired a carbapenemsusceptible or non-susceptible K. pneumoniae strain, respectively, during their ICU stay (Supplementary Table 5, adjusted Odds Ratio [aOR]: 0.40 [99\% CI: 0.14-1.13], $p=0.023$ and 1.03 [0.36-2.97], $p=0.937$ ). Interestingly, the group of patients that carried a carbapenemsusceptible strain of $K$. pneumoniae, either on admission or acquired during ICU stay, had the lowest observed 


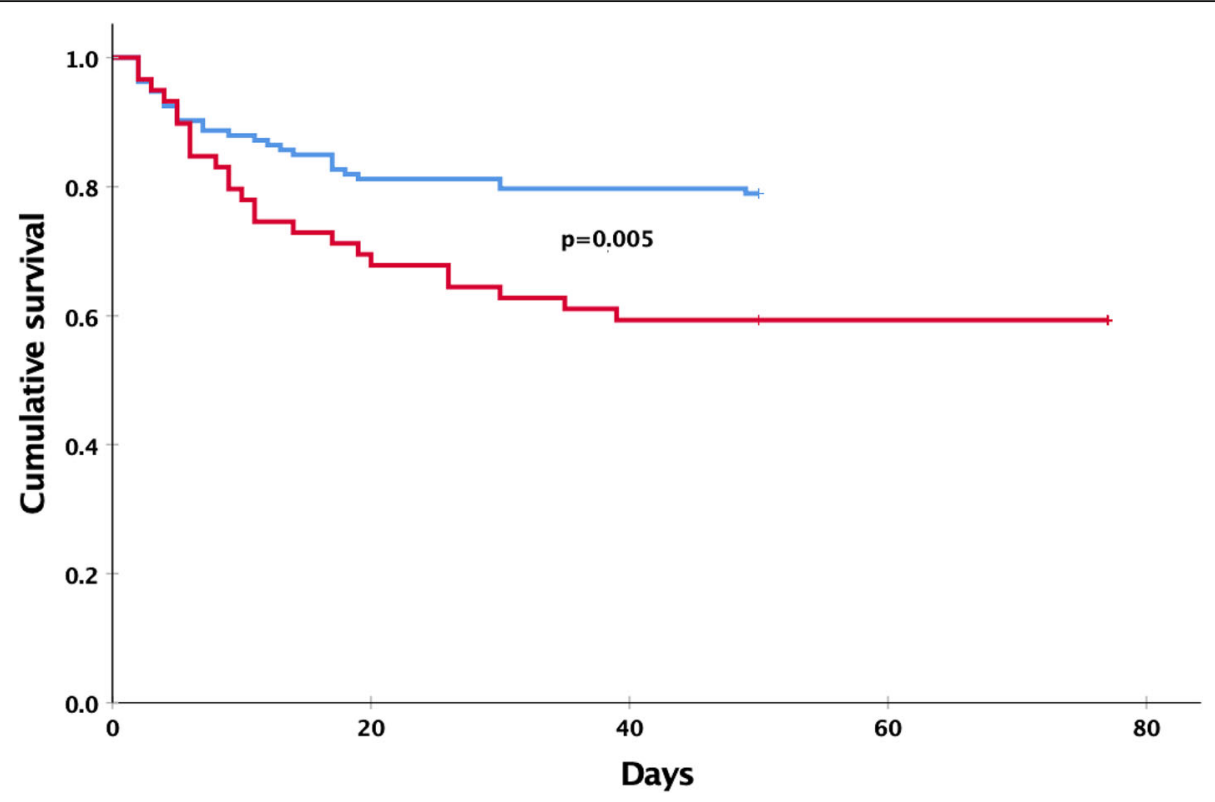

Fig. 3 Survival of patients according to their K. pneumoniae status. Survival of patients with carbapenem-non-susceptible K. pneumoniae (on admission or acquired during ICU stay) (red line) compared with the survival of patients that had carbapenem-susceptible K. pneumoniae (on admission or acquired during ICU stay) (blue line) in their screening and/or clinical cultures. $P$ value was calculated using logistic regression

mortality rates (24.3 and $17.5 \%$, respectively), even lower than the $30.5 \%$ mortality observed among those patients that were always negative for this species, but this difference did not reach statistical significance. However, when compared to patients that had a carbapenem-nonsusceptible isolate of $K$. pneumoniae, either on admission or during their ICU stay, the ICU survival of patients with carbapenem-susceptible strains was significantly higher (aHR: 2.57 [99\% CI: 1.07-6.17], $p=0.005$, Fig. 3). Importantly, the admission SIRS and qSOFA scores of patients with or without $K$. pneumoniae acquisition did not differ (Table 1), indicating that a difference in the risk of dying was not present at the time of ICU admission but emerged later during their ICU stay (SIRS: crude Odds Ratio [cOR]: 1.69 [99\% CI: 0.55-5.22], $p=0.230$; qSOFA: cOR: 1.45 [99\% CI: $0.68-3.08], \quad p=0.211$, Supplementary Table 5).

The competing risk estimates analysis also revealed that the incidence of death was higher in patients with a carbapenem-non-susceptible isolate of $K$. pneumoniae $(p=0.006)$, and the incidence of being discharged alive from ICU was higher for patients with a carbapenem-susceptible $K$. pneumoniae $(p=0.0005)$ (Supplementary Figure 2). Patients that acquired a carbapenem-non-susceptible $K$. pneumoniae during ICU stay were more likely to have had prior exposure to antibiotics, especially carbapenems, and they were more likely to have had a medical indication for their admission to the ICU (Table 1).

\section{Phenotypic and molecular characterization of carbapenem-non-susceptible $K$. pneumoniae}

Overall, 99/370 (26.8\%) isolates from 59/192 (30.7\%) patients were found to be non-susceptible to carbapenems. In addition, one (water from suction connector) out of $31 \mathrm{~K}$. pneumoniae isolates cultured from the environment (400 samples taken) was carbapenem-nonsusceptible. None of $24 \mathrm{~K}$. pneumoniae isolates cultured from HCWs (out of 167 screened) were found to be carbapenem-non-susceptible. Thus, a total of 100 carbapenem-non-susceptible isolates was further subjected to phenotypic and molecular analyses. The phenotypic detection test indicated that $96 / 100$ (96\%) isolates produced a MBL. PCRs of carbapenemase genes demonstrated the presence of the $b l a_{\mathrm{NDM}}$ gene in these 96 carbapenem-non-susceptible isolates, including isolates from patients and the one from the environment. None of the 100 isolates was positive for either the $b l a_{\mathrm{KPC}}$ or $b l a_{\text {OXA-48 }}$ gene. Four carbapenem-non-susceptible strains apparently contained another resistance mechanism, which was not further investigated, they remained relatively susceptible to carbapenems (MIC meropenem $2-4 \mathrm{mg} / \mathrm{L}$ ). The string test was positive for only four isolates from three patients, one of whom deceased in ICU.

\section{Clonal relatedness}

Raman spectroscopy analysis performed for 100 isolates revealed the presence of multiple types within 
Table 1 Patient characteristics and outcomes according to their Klebsiella pneumoniae status

\begin{tabular}{|c|c|c|c|c|c|c|}
\hline & Group 1 & Group 2 & Group 3 & Group 4 & Group 5 & $p$ value \\
\hline & 220 & 70 & 22 & 63 & 37 & \\
\hline Age (years), median (IQR) & $46(33-58)$ & $49(33-58)$ & $31.5(25-49)$ & $50(38-58)$ & $47(35.5-62)$ & 0.036 \\
\hline Gender (\%) & & & & & & 0.533 \\
\hline Male & $108(49.1)$ & $35(50)$ & $13(59.1)$ & $35(55.6)$ & $23(62.2)$ & \\
\hline Female & $112(50.9)$ & $35(50)$ & $9(40.9)$ & $28(44.4)$ & $14(37.8)$ & \\
\hline \multicolumn{7}{|l|}{ Underlying diseases (\%) } \\
\hline Cardiovascular & & & & & & 0.556 \\
\hline Yes & $10(4.5)$ & $64(91.4)$ & $1(4.5)$ & $5(7.9)$ & $3(8.1)$ & \\
\hline No & $210(95.5)$ & $6(8.6)$ & $21(95.5)$ & $58(92.1)$ & $34(91.9)$ & \\
\hline Cerebrovascular & & & & & & 0.026 \\
\hline Yes & $11(5.0)$ & $2(2.9)$ & $3(13.6)$ & $8(12.7)$ & $5(13.5)$ & \\
\hline No & $209(95.0)$ & $68(97.1)$ & 19 (86.4) & $55(87.3)$ & $32(86.5)$ & \\
\hline Chronic kidney disease & & & & & & 0.068 \\
\hline Yes & $17(7.7)$ & $7(10.0)$ & 0 & $1(1.6)$ & 0 & \\
\hline No & $203(92.3)$ & $63(70.0)$ & $22(100)$ & $62(98.4)$ & $37(100.0)$ & \\
\hline Diabetes mellitus & & & & & & 0.138 \\
\hline Yes & $12(5.5)$ & $10(14.3)$ & $1(4.5)$ & $6(9.5)$ & $4(10.8)$ & \\
\hline No & $208(94.5)$ & $60(85.7)$ & $21(95.5)$ & $57(90.5)$ & $33(89.2)$ & \\
\hline Malignancy & & & & & & 0.717 \\
\hline Yes & $68(30.9)$ & $20(28.6)$ & $4(18.2)$ & $16(25.4)$ & $10(27.0)$ & \\
\hline No & $152(69.1)$ & $50(71.4)$ & $18(81.8)$ & $47(74.6)$ & $27(73.0)$ & \\
\hline Indication for ICU admission (\%) & & & & & & 0.005 \\
\hline Medical & $64(29.1)$ & $21(30.0)$ & $10(45.5)$ & $23(36.5)$ & $22(59.5)$ & \\
\hline Surgical & $156(70.9)$ & $49(70.0)$ & $12(54.5)$ & $40(63.5)$ & $15(40.5)$ & \\
\hline Referral from (\%) & & & & & & 0.378 \\
\hline Other ward this hospital & $115(52.3)$ & $36(51.4)$ & $15(68.2)$ & $33(52.4)$ & $23(62.2)$ & \\
\hline Other hospital & $40(18.2)$ & $14(20.0)$ & $2(9.1)$ & $11(17.5)$ & $10(27.0)$ & \\
\hline Directly from Emergency Unit & $65(29.5)$ & $20(28.6)$ & $5(22.7)$ & $19(30.2)$ & $4(10.8)$ & \\
\hline \multicolumn{7}{|c|}{ Antibiotic exposure (pre-ICU admission) } \\
\hline Any antibiotic (\%) & $163(74.1)$ & $54(77.1)$ & $18(81.8)$ & $44(69.8)$ & $32(86.5)$ & 0.365 \\
\hline Carbapenem (\%) & $44(20.0)$ & $3(4.3)$ & $7(31.8)$ & $12(19.0)$ & $13(35.1)$ & $<0.001$ \\
\hline SIRS Score, (\%) & & & & & & 0.598 \\
\hline Score $>2$ & $200(91.0)$ & $63(70.0)$ & $22(100.0)$ & $59(93.7)$ & $33(89.2)$ & \\
\hline Score $<2$ & $20(9.0)$ & $7(10.0)$ & 0 & $4(6.3)$ & $4(10.8)$ & \\
\hline qSOFA Score, (\%) & & & & & & 0.971 \\
\hline Score $\geq 2$ & $179(81.4)$ & $56(80.0)$ & $17(77.3)$ & $51(81.0)$ & $31(83.2)$ & \\
\hline Score $<2$ & $41(18.6)$ & $14(20.0)$ & $5(22.7)$ & $12(19.0)$ & $6(16.2)$ & \\
\hline \multicolumn{7}{|l|}{ Procedures (during ICU admission) } \\
\hline Mechanical ventilation used (\%) & $199(90.5)$ & $59(84.3)$ & $20(90.9)$ & $57(90.5)$ & $36(97.3)$ & 0.314 \\
\hline Mechanical ventilation (days) & & & & & & $<0.001$ \\
\hline$\geq 5$ days & $74(33.6)$ & $30(42.9)$ & $12(54.5)$ & $34(54.0)$ & $32(86.5)$ & \\
\hline$<5$ days & $146(66.4)$ & $40(57.1)$ & $10(45.5)$ & $29(46.0)$ & $5(13.5)$ & \\
\hline Central venous catheter used (\%) & $193(87.7)$ & $62(88.6)$ & $19(86.4)$ & $53(84.1)$ & $36(97.3)$ & 0.343 \\
\hline
\end{tabular}


Table 1 Patient characteristics and outcomes according to their Klebsiella pneumoniae status (Continued)

\begin{tabular}{|c|c|c|c|c|c|c|}
\hline & Group 1 & Group 2 & Group 3 & Group 4 & Group 5 & $p$ value \\
\hline & 220 & 70 & 22 & 63 & 37 & \\
\hline Central venous catheter (days) & & & & & & $<0.001$ \\
\hline$\geq 5$ days & $96(43.6)$ & $40(57.1)$ & $12(54.5)$ & $42(66.7)$ & $33(89.2)$ & \\
\hline$<5$ days & $124(56.4)$ & $30(42.9)$ & $10(45.5)$ & $21(33.3)$ & $4(10.8)$ & \\
\hline Urinary catheter (\%) & $220(100.0)$ & $70(100.0)$ & $22(100.0)$ & $63(100.0)$ & $37(100.0)$ & NA \\
\hline \multicolumn{7}{|l|}{ Urinary catheter (days) median (IQR) } \\
\hline$\geq 5$ days & $112(50.9)$ & $44(62.9)$ & $15(68.2)$ & $46(73.0)$ & $34(91.9)$ & $<0.001$ \\
\hline$<5$ days & $108(49.1)$ & $26(37.1)$ & $7(31.8)$ & $17(27.0)$ & $3(8.1)$ & \\
\hline \multicolumn{7}{|l|}{ Antibiotic therapy (during ICU admission) } \\
\hline Any antibiotic (\%) & $217(98.2)$ & $70(100.0)$ & $21(95.5)$ & $62(98.4)$ & $36(100.0)$ & 0.474 \\
\hline Carbapenem (\%) & $110(49.8)$ & $26(37.1)$ & $13(59.1)$ & $29(46.0)$ & $21(58.3)$ & 0.179 \\
\hline \multicolumn{7}{|l|}{ Outcomes } \\
\hline Length of stay (days), median (IQR) & $4(2-6)$ & $5(3-9)$ & $6(3-13)$ & $7(4-13)$ & $11(8-20)$ & $<0.001$ \\
\hline Death (\%) & $67(30.3)$ & $17(24.3)$ & $8(36.4)$ & $11(17.5)$ & $16(44.4)$ & 0.054 \\
\hline
\end{tabular}

Abbreviations: ICU Intensive Care Unit; IQR Interquartile range; NS Non-Susceptible; qSOFA quick Sepsis-related Organ Failure Assessment; SIRS Systemic Inflammatory Response Syndrome; S Susceptible

Group 1: No K. pneumoniae on admission and negative for $K$. pneumoniae during ICU admission

Group 2: Carbapenem-S K. pneumoniae on admission, no carbapenem-NS K. pneumoniae acquisition during ICU admission

Group 3: Carbapenem-NS K. pneumoniae on admission, considered as positive during ICU admission (regardless of results of follow-up cultures)

Group 4: No K. pneumoniae on admission, acquisition of carbapenem-S K. pneumoniae during ICU admission

Group 5: Either no K. pneumoniae or carbapenem-S K. pneumoniae on admission, acquisition of carbapenem-NS K. pneumoniae during ICU admission

Significance was calculated using One-way ANOVA, Pearson Chi Square and Fisher's Exact Test

A $p$-value less than 0.01 was considered statistically significant

this collection of $K$. pneumoniae. In total, 48 Raman types were identified. There were three major clusters (Supplementary Figure 3), the largest cluster (CIPTOKPN24) consisted of 20 isolates obtained from 13 patients (screening and clinical specimens). Strains belonging to the dominant cluster CIPTOKPN24 were present in both ICUs throughout the study period, whereas other clones seemed to wax and wane with time (Fig. 4).

A total of 97 clinical (two isolates were lost during storage) and 1 environmental isolate were further analyzed using MLVA genotyping, identifying 30 different genotypes (Fig. 5). The most dominant clone accounted for $26.5 \%(n=26)$ of all isolates, whereas 19 isolates (19.4\%) were of a unique genotype, the remaining 53 isolates belonged to 20 other genotypes. Clustering of strains by Raman spectroscopy into three dominant groups was concordant with clustering by MLVA, e.g. the 20 Raman CIPTOKPN24 strains all belonged to a single MLVA clonal complex. Likewise, the four Raman CIPTOKPN30 strains belonged to a single MLVA clonal complex as did 8/ 10 CIPTOKPN27 isolates.

\section{Discussion}

This is the first report of a study on the clinical and molecular epidemiology of carbapenemase-producing
K. pneumoniae in ICUs in Indonesia. These two ICUs can be considered to have endemic carbapenem-nonsusceptible $K$. pneumoniae whose acquisition by patients is associated with prolonged ICU stay and, possibly, an increased risk of dying.

The dissemination of $K$. pneumoniae isolates harboring carbapenemase genes, continues unabated, and reports describing these isolates are emerging from different parts of the world, including Southeast Asia [1, 4, 28]. Colonization and infection with carbapenem-resistant $K$. pneumoniae has been reported in Singapore [29]. In Malaysia, the National Surveillance of Antimicrobial resistance found carbapenem resistance rates among $K$. pneumoniae to increase from $0.5 \%$ (11,935 isolates tested) in 2010 to $1.6 \%$ (27,911 isolates tested) in 2014 [30]. The Philippines Department of Health's Research Institute reported a rate of $11.9 \%$ in 2015 [30]. Morocco [31], Italy [32], and India have likewise shown dramatic increases over time [30]. Similar to these studies, we found that 59/ $412(14.3 \%)$ of the patients in the ICU carried a carbapenem-non-susceptible K. pneumoniae. By screening on ICU admission $5.3 \%$ of patients were already colonized with carbapenem-non-susceptible $K$. pneumoniae prior to their admission to the ICU. This suggests that patients may become colonized with such strains elsewhere in the same hospital or in another hospital from which they are referred, or may come with such strain directly from the 

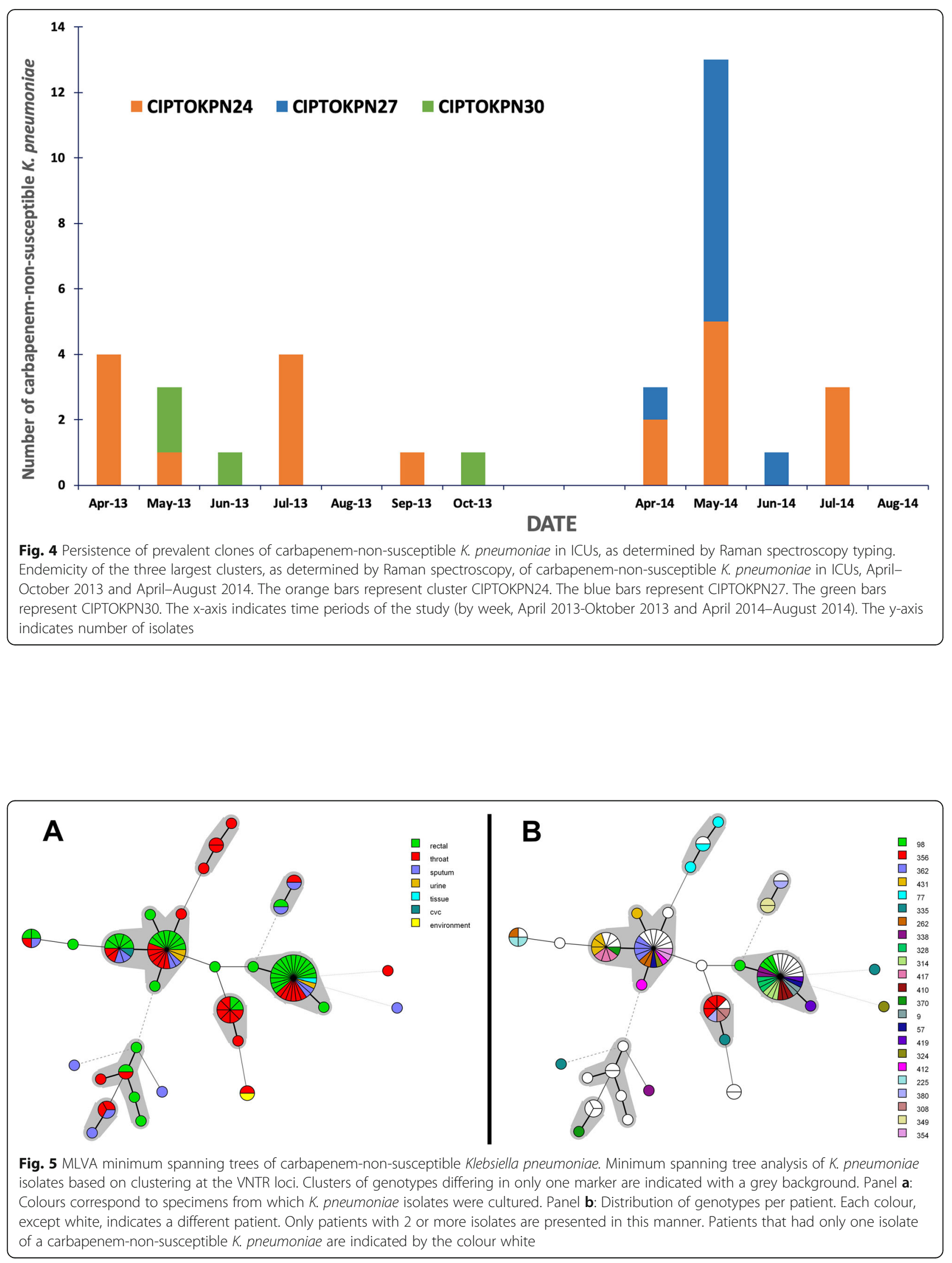
community, possibly having acquired their strain during a previous healthcare contact or indirectly from exposure to reservoirs or relatives carrying such strains [33].

Screening cultures can, therefore, be considered helpful for early detection and infection control. It may also be useful to guide rational antibiotic use, since previous studies have shown that colonization with a carbapenem-resistant $K$. pneumoniae is a risk factor for subsequent infection [34, 35]. However, during our study, carbapenem-non-susceptible $K$. pneumoniae strains were not isolated from blood cultures (data not shown).

Our data also show that patients may acquire carbapenem-non-susceptible $K$. pneumoniae during ICU stay in the setting of our study and that these acquisitions are associated with significantly longer ICU stay. At the level of significance chosen, the acquisition of $K$. pneumoniae strains, whether carbapenem susceptible or not, was not associated with mortality when compared to patients who remained free of $K$. pneumoniae. In contrast, the study from Dautzenberg et al. (2015) showed patients colonized with carbapenemase-producing Enterobacteriaceae to have on average a 1.79 times higher hazard of dying in ICU than non-colonized patients, primarily because of an increased length of stay [36]. A study in Singapore reported that cases with carbapenemresistant strains of Enterobacteriaceae had $~ 3.5$ times increased odds of fatality adjusted for length of hospital stay [29].

Interestingly, our study shows that the risk of dying among ICU patients who were culture-positive for carbapenem-non-susceptible $K$. pneumoniae on admission or during ICU stay was significantly, 2.57 times, higher than among patients who were culturepositive for a carbapenem-susceptible $K$. pneumoniae. The observed ICU fatality rates were indeed highest (44.4\%) among those acquiring carbapenemnon-susceptible $K$. pneumoniae and lowest (17.5\%) amongst the patients acquiring carbapenemsusceptible strains of $K$. pneumoniae during their ICU stay. Since risk of mortality during ICU stay is influenced by many factors, as reported by other international findings [32, 37, 38], we cannot readily explain why susceptible $K$. pneumoniae acquisitions seem to be a proxy for protection whereas nonsusceptible $K$. pneumoniae acquisitions may be predictive of a fatal outcome. Probably, exposure to carbapenem antibiotics, and the underlying reasons for this, may be important determinants in this respect.

The $b l a_{\mathrm{NDM}}$ gene was the most prevalent carbapenemase gene as it was detected in 96 isolates, including one from the environment. Carbapenem resistance due to $b l a_{\mathrm{KPC}}-$ like and $b l a_{\mathrm{OXA}-48}$-like genes was not detected.
In South Asia (India and Pakistan) the NDM-1 gene was initially found and currently, this enzyme is by far the most prevalent and widely distributed carbapenem degrading enzyme in the world, including in Southeast Asia [1, 3-6, 8-10, 18, 28-31].

Walsh et al. (2011) have also found the presence of NDM-1 $\beta$-lactamase-producing bacteria, including K. pneumoniae, from waste seepages samples in Indian community [33]. Taking our study and the recent report on NDM-1 in carbapenem-non-susceptible Enterobacteriaceae from urinay tract samples of hospitalized patients in Surabaya, Indonesia, into account, we suspect that this carbapenemase gene is widespread in hospitals in Indonesia [39].

Carbapenem-susceptible and -non-susceptible $K$. pneumoniae that colonize or infect ICU patients may originate from the patient her/himself, but may also come from contaminated hospital equipment and environment, staff and other patients. In this study $K$. pneumoniae was found in the ICU environment, including one endemic strain that was carbapenemnon-susceptible. Predictably, K. pneumoniae was also cultured from throat and rectal swabs of ICU personnel, although none of those isolates was carbapenem-non-susceptible. However, we cannot exclude personnel as a source or vector of $K$. pneumoniae since personnel was only screened once during this study and other body parts (e.g. hands) or clothes were not sampled, limiting the sensitivity of this part of the survey. A recent study in China found that almost $9 \%$ of medical personnel in ICU carried multidrug-resistant Gram-negative bacteria on their hands [40]. Transmission of the bacteria may occur with many risk factors involved $[1,3,41]$. Multiple studies reported outbreaks of carbapenemresistant $K$. pneumoniae that were associated with environmental contamination [42-44]. We performed Raman spectroscopy and MLVA to assess clonal relatedness. These analyses revealed three major clusters by Raman typing, with the largest one (CIPTOKPN24), persisting in both ICUs throughout the whole study period. However, many carbapenemnon-susceptible strains of $K$. pneumoniae cultured in this study were of a unique Raman type or belonged to small clusters that waxed and waned quickly, indicating both endemicity of certain clones in the ICUs but also regular new introductions and rapid loss of many clones over time. This epidemiologic information can and should be applied when designing interventions to reduce the acquisition of carbapenemresistant $K$. pneumoniae in ICUs in Indonesia and in similar settings elsewhere.

Although not used routinely, Raman spectroscopy typing can be valuable for discriminating types of 
strains within a species [21-23]. Here we showed typing by Raman spectroscopy to yield $K$. pneumoniae strain clustering compatible with clustering based on MLVA genotyping. However, Raman typing results at a given site cannot be directly compared with results generated or published elsewhere, and are, thus, not easily shared or pooled. Considering the modification that we made to MLVA marker VNTR58, the genotype of the most dominant clone in our study was of genotype "4-2.4-3-4-4.3-1-1219". This genotype would translate into "4-3-3-4-51-12-19" based on the original MLVA typing method for $K$. pneumoniae [24], but this genotype was not observed by Brink et al. [24]. On the other hand, the second and third most dominant genotype "5-3-3-46-1-9-12" and "5-3-3-5-6-1-9-12" match genotype "5-3-3-4-6-1-9-1" and a single locus variant thereof from Brink et al. that involves isolates with MLST sequence type ST147. K. pneumoniae ST147 strains belong to a relatively common NDM-positive $K$. pneumoniae lineage and have been found in multiple countries across several continents, almost all of which were isolated from humans $[45,46]$. Unlike in the original paper, in our approach the non-integer alleles were considered as separate alleles. As a result, the total number of alleles per marker will increase for several markers and as a result this may benefit the overall discriminatory power of the MLVA method. Indeed, in our study (exceeding the ones reported here), we observed several different genotypes that would have been assigned identical when the non-integer alleles would have been ignored by 'rounding off' their values using adjusted and broadened binsets (results not shown).

There were some limitations in this study. First, as no colometric agar plate was used for the screening cultures, overgrowth of carbapenem-susceptible but cefotaxime-resistant isolates could have led to overlooking CNKP. However, all morphologically different colonies were checked, and this was done by trained and experienced microbiology technicians. Colometric media are expensive, therefore, could not be used in this study. Also, cefotaxime-susceptible OXA-48-producing isolates could have been missed with our screening method. Nevertheless, based on the isolates found in the clinical cultures, it is unlikely that these were playing a major role in the epidemiology. Of note, the OXA-48 PCR was not able to detect OXA-54 and OXA-436, but given the results of the phenotypic detection method, and epidemiology in countries nearby, these are also not suspected. Second, only two ICUs in one tertiary care academic hospital participated, which does not permit results to be called representative for all
ICUs in Indonesia. Third, the study was performed more than 5 years ago, hence the epidemiology of CNKP in Indonesia may be different now. Finally, the limited rate of sampling of the environment and of personnel (as opposed to patients) may have undervalued their role in the chain of transmission and acquisition of carbapenem-non-susceptible $K$. pneumoniae.

\section{Conclusions}

In summary, this study is the largest to date that describes the characteristics and epidemiology of, and outcome associated with carbapenem-non-susceptible K. pneumoniae in ICUs in Indonesia. Colonization or infection with carbapenem-non-susceptible $K$. pneumoniae during hospitalization was independently associated with prolonged LOS in the ICU, and may affect survival during ICU stay. Prevention of colonization by and infection from these multidrugresistant strains requires interventions directed to source control and limiting the introduction and transmission of such strains to and between patients.

\section{Supplementary information}

Supplementary information accompanies this paper at https://doi.org/10. 1186/s13756-020-00716-7.

\section{Additional file 1: Table S1. List of environmental samples. Table S2. Amplification primers used for MLVA typing. Modification of the original MLVA typing method of Brink et al. Table S3. Baseline characteristics of 412 patients admitted to the adult or Emergency Room (ER) ICUs, and enrolled in this study. Table S4. Variables associated with length of stay among patients with and without carbapenem-non-susceptible Klebsiella pneumoniae. Table S5. Variables associated with mortality among pa- tients with and without carbapenem-non-susceptible Klebsiella pneumoniae. \\ Additional file 2: Figure S1. Klebsiella pneumoniae carriage of included patients admitted to the ICUs (adult- and ER-ICU) of Dr. Cipto Mangunkusomo General Hospital, Jakarta, Indonesia. Figure S2. Plot of the cumulative incidence for ICUs discharge alive and death by carbapenem-susceptible and -non-susceptible K. pneumoniae. Figure S3. Raman spectroscopy-based cluster analysis of Klebsiella pneumoniae iso- lates from adult- and ER-ICUs.}

\begin{abstract}
Abbreviations
aHR: Adjusted Hazard Ratio; aOR: Adjusted Odds Ratio; ATCC: American Type Culture Collection; Cl: Confidence interval; CL: Cloxacillin; CNKP: Carbapenemnon-susceptible Klebsiella pneumoniae; cOR: Crude Odds Ratio;

CRAN: Comprehensive R Archive Network; DPA: Dipicolinic acid;

ER: Emergency Room; EUCAST: European Committee on Antimicrobial Susceptibility Testing; HCW: Healthcare worker; ICU: Intensive care unit; IMP: Imipenemase; IQR: Interquartile range; KPC: Klebsiella pneumoniae carbapenemase; LOS: Length of stay; MBL: Metallo- $\beta$-lactamase;

MIC: Minimum inhibitory concentration; MLVA: Multiple-locus variable number tandem repeat analysis; NDM: New Delhi metallo- $\beta$-lactamase; OXA: Oxacillinase; PBA: Phenyl boronic acid; qSOFA: Quick Sequential Organ Failure Assessment; SIRS: Systemic inflammatory response syndrome;

TSB: Trypticase soy broth; VIM: Verona integron-encoded metallo- $\beta$-lactamase
\end{abstract}

\section{Acknowledgements}

We are thankful to The Directorate General of Higher Education of Indonesia Ministry of Research, Technology and Higher Education of the Republic of 
Indonesia, Dean of Faculty of Medicine Universitas Indonesia, Board of Directors of Dr. Cipto Mangunkusumo National General Hospital Jakarta Indonesia, Department of Medical Microbiology and Infectious Diseases, especially Michelle de Regt and Liesbeth van der Zwaan for their support in the laboratory, Erasmus MC in Rotterdam, The Netherlands and Critical Care Division, Department of Anesthesia and Intensive Care, Faculty of Medicine Universitas Indonesia / Dr. Cipto Mangunkusumo General Hospital, Jakarta, Indonesia.

\section{Ethics approval and consent to participate}

Informed consent was documented by the use of a written consent formapproved by the Ethics Committee Faculty of Medicine Universitas Indonesia/ Dr. Cipto Mangunkusumo General Hospital and signed and dated by thesubjects/guardians and by the person who conducted the informed consentdiscussion and two witnesses. The signature confirmed the consent wasbased on information that had been understood.

- The Ethics Committee of the Faculty of Medicine, Universitas Indonesia, approved the research on 17th September 2012, No: 561/ PT02.FK/ETIK/2012, No: 757/UN2.F1/ETIK/X/2014.

- A Material Transfer Agreement (MTA) was reviewed and approved by the Director of National Institute Research and Development, Ministry of Health (No: LB.02.01/I.9.4/8500/2013).

\section{Authors' contributions}

YRS, AK, HAV, and JAS conceived the study and participated in design of the study. YRS, RS, and DA participated in acquisition of data. YRS, WHFG, CHWK, HAV, and JAS performed data analysis and interpreted the data. YRS, CHWK, $H A V$, and JAS drafted the article. All authors participated in critically revising the draft. All authors read and approved the final manuscript.

\section{Funding}

This work was supported by The Directorate General of Higher Education of Indonesia Ministry of Research, Technology and Higher Education of the Republic of Indonesia' and 'Department of Medical Microbiology and Infectious Diseases, Erasmus MC in Rotterdam, The Netherlands'.

\section{Availability of data and materials}

The datasets used and/or analysed during the current study are available from the corresponding author on reasonable request.

\section{Competing interests}

YRS is an awardee of the DIKTI-NESO Scholarship by The Directorate General of Higher Education of Indonesia Ministry of Research, Technology and Higher Education of the Republic of Indonesia, and Department of Medical Microbiology and Infectious Diseases, Erasmus MC in Rotterdam, The Netherlands. All authors report no conflict of interest relevant to this article.

\section{Author details}

${ }^{1}$ Department of Clinical Microbiology, Faculty of Medicine, Universitas Indonesia / Dr. Cipto Mangunkusumo General Hospital, Jakarta, Indonesia. ${ }^{2}$ Department of Medical Microbiology and Infectious Diseases, Erasmus MC University Medical Center Rotterdam, Dr. Molewaterplein 40, 3015, GD, Rotterdam, The Netherlands. ${ }^{3}$ Critical Care Division, Department of Anesthesia and Intensive Care, Faculty of Medicine, Universitas Indonesia / Dr. Cipto Mangunkusumo General Hospital, Jakarta, Indonesia.

\section{Received: 3 January 2020 Accepted: 2 April 2020}

\section{Published online: 11 May 2020}

\section{References}

1. Lee CR, Lee JH, Park KS, Kim YB, Jeong BC, Lee SH. Global dissemination of carbapenemase-producing Klebsiella pneumoniae: epidemiology, genetic context, treatment options, and detection methods. Front Microbiol. 2016;7:895.

2. Munoz-Price LS, Poirel L, Bonomo RA, Schwaber MJ, Daikos GL, Cormican M, et al. Clinical epidemiology of the global expansion of Klebsiella pneumoniae carbapenemases. Lancet Infect Dis. 2013;13(9):785-96.

3. Queenan AM, Bush K. Carbapenemases: the versatile beta-lactamases. Clin Microbiol Rev. 2007;20(3):440-58.
4. Tängdén T, Giske CG. Global dissemination of extensively drug-resistant carbapenemase-producing Enterobacteriaceae: clinical perspectives on detection, treatment and infection control. J Intern Med. 2015;277(5):501-12.

5. Nordmann P. Carbapenemase-producing Enterobacteriaceae: overview of a major public health challenge. Med Mal Infect. 2014;44(2):51-6.

6. Shakil S, Azhar El, Tabrez S, Kamal MA, Jabir NR, Abuzenadah AM, et al. New Delhi metallo-beta-lactamase (NDM-1): an update. J Chemother. 2011;23(5): 263-5.

7. Dortet L, Cuzon G, Nordmann P. Dissemination of carbapenemaseproducing Enterobacteriaceae in France, 2012. J Antimicrob Chemother. 2014;69(3):623-7.

8. Dortet L, Poirel L, Nordmann P. Worldwide dissemination of the NDM-type carbapenemases in gram-negative bacteria. Biomed Res Int. 2014;2014: 249856.

9. Teo J, Ngan G, Balm M, Jureen R, Krishnan P, Lin R. Molecular characterization of NDM-1 producing Enterobacteriaceae isolates in Singapore hospitals. Western Pac Surveill Response J. 2012;3(1):19-24.

10. Rimrang B, Chanawong A, Lulitanond A, Wilailuckana C, Charoensri N, Sribenjalux $\mathrm{P}$, et al. Emergence of NDM-1- and IMP-14a-producing Enterobacteriaceae in Thailand. J Antimicrob Chemother. 2012;67(11): 2626-30.

11. Karuniawati A, Saharman YR, Lestari DC. Detection of carbapenemase encoding genes in Enterobacteriaceae, Pseudomonas aeruginosa, and Acinetobacter baumannii isolated from patients at intensive care unit Cipto Mangunkusumo hospital in 2011. Acta Med Indones. 2013;45(2):101-6.

12. Sugianli AK, Ginting F, Kusumawati RL, Pranggono EH, Pasaribu AP, Gronthoud F, et al. Antimicrobial resistance in uropathogens and appropriateness of empirical treatment: a population-based surveillance study in Indonesia. J Antimicrob Chemother. 2017;72(5):1469-77.

13. Singer M, Deutschman CS, Seymour CW, Shankar-Hari M, Annane D, Bauer $M$, et al. The third international consensus definitions for Sepsis and septic shock (Sepsis-3). JAMA. 2016;315(8):801-10.

14. Saharman YR, Karuniawati A, Sedono R, Aditianingsih D, Sudarmono P, Goessens WHF, et al. Endemic carbapenem-nonsusceptible Acinetobacter baumannii-calcoaceticus complex in intensive care units of the national referral hospital in Jakarta, Indonesia. Antimicrob Resist Infect Control. 2018;7:5.

15. The European Committee on Antimicrobial Susceptibility Testing. Breakpoint tables for interpretation of MICs and zone diameters. Version 3.1 [Internet]. 2013. Available from: http://www.eucast.org.

16. Nadasy KA, Domiati-Saad R, Tribble MA. Invasive Klebsiella pneumoniae syndrome in North America. Clin Infect Dis. 2007;45(3):e25-8.

17. Van Dijk K, Voets GM, Scharringa J, Voskuil S, Fluit AC, Rottier WC, et al. A disc diffusion assay for detection of class A, B and OXA-48 carbapenemases in Enterobacteriaceae using phenyl boronic acid, dipicolinic acid and temocillin. Clin Microbiol Infect. 2014;20(4):345-9.

18. Islam MA, Talukdar PK, Hoque A, Huq M, Nabi A, Ahmed D, et al. Emergence of multidrug-resistant NDM-1-producing gram-negative bacteria in Bangladesh. Eur J Clin Microbiol Infect Dis. 2012;31(10): 2593-600.

19. Moland ES, Black JA, Ourada J, Reisbig MD, Hanson ND, Thomson KS. Occurrence of newer beta-lactamases in Klebsiella pneumoniae isolates from 24 U.S. hospitals. Antimicrob Agents Chemother. 2002;46(12):3837-42.

20. Aktas Z, Kayacan CB, Schneider I, Can B, Midilli K, Bauernfeind A. Carbapenem-hydrolyzing oxacillinase, OXA-48, persists in Klebsiella pneumoniae in Istanbul, Turkey. Chemotherapy. 2008:54(2):101-6.

21. Maquelin K, Dijkshoorn L, van der Reijden TJ, Puppels GJ. Rapid epidemiological analysis of Acinetobacter strains by Raman spectroscopy. J Microbiol Methods. 2006;64(1):126-31.

22. Willemse-Erix DF, Scholtes-Timmerman MJ, Jachtenberg JW, van Leeuwen WB, Horst-Kreft D, Bakker Schut TC, et al. Optical fingerprinting in bacterial epidemiology: Raman spectroscopy as a real-time typing method. J Clin Microbiol. 2009;47(3):652-9.

23. Willemse-Erix D, Bakker-Schut T, Slagboom-Bax F, Jachtenberg JW, Lemmens-den Toom N, Papagiannitsis CC, et al. Rapid typing of extendedspectrum beta-lactamase- and carbapenemase-producing Escherichia coli and Klebsiella pneumoniae isolates by use of SpectraCell RA. J Clin Microbiol. 2012;50(4):1370-5

24. Brink AA, von Wintersdorff CJ, van der Donk CF, Peeters AM, Beisser PS, Stobberingh $\mathrm{EE}$, et al. Development and validation of a single-tube multiple-locus variable number tandem repeat analysis for Klebsiella pneumoniae. PLoS One. 2014;9(3):e91209. 
25. Gray RJ. A class of K-sample tests for comparing the cumulative incidence of a competing risk. Ann Stat. 1988;16(3):1141-54.

26. Scrucca L, Santucci A, Aversa F. Competing risk analysis using R: an easy guide for clinicians. Bone Marrow Transpl. 2007:40(4):381-7.

27. Johnson VE. Revised standards for statistical evidence. Proc Natl Acad Sci U S A. 2013:110(48):19313-7.

28. Nordmann P, Dortet L, Poirel L. Carbapenem resistance in Enterobacteriaceae: here is the storm! Trends Mol Med. 2012;18(5):263-72.

29. Ling ML, Tee YM, Tan SG, Amin IM, How KB, Tan KY, et al. Risk factors for acquisition of carbapenem resistant Enterobacteriaceae in an acute tertiary care hospital in Singapore. Antimicrob Resist Infect Control. 2015;4:26.

30. Hsu LY, Apisarnthanarak A, Khan E, Suwantarat N, Ghafur A, Tambyah PA. Carbapenem-resistant Acinetobacter baumannii and Enterobacteriaceae in south and Southeast Asia. Clin Microbiol Rev. 2017;30(1):1-22.

31. Barguigua A, Zerouali K, Katfy K, El Otmani F, Timinouni M, Elmdaghri N. Occurrence of OXA-48 and NDM-1 carbapenemase-producing Klebsiella pneumoniae in a Moroccan university hospital in Casablanca. Morocco Infect Genet Evol. 2015;31:142-8.

32. Hoxha A, Karki T, Giambi C, Montano C, Sisto A, Bella A, et al. Attributable mortality of carbapenem-resistant Klebsiella pneumoniae infections in a prospective matched cohort study in Italy, 2012-2013. J Hosp Infect. 2016; 92(1):61-6.

33. Walsh TR, Weeks J, Livermore DM, Toleman MA. Dissemination of NDM-1 positive bacteria in the New Delhi environment and its implications for human health: an environmental point prevalence study. Lancet Infect Dis. 2011;11(5):355-62

34. Martin RM, Cao J, Brisse S, Passet V, Wu W, Zhao L, et al. Molecular Epidemiology of Colonizing and Infecting Isolates of Klebsiella pneumoniae. mSphere. 2016;1(5):e00261-16:1-1212.

35. Borer A, Saidel-Odes L, Eskira S, Nativ R, Riesenberg K, Livshiz-Riven I, et al. Risk factors for developing clinical infection with carbapenem-resistant Klebsiella pneumoniae in hospital patients initially only colonized with carbapenem-resistant K. pneumoniae. Am J Infect Control. 2012;40(5):421-5.

36. Dautzenberg MJ, Wekesa AN, Gniadkowski M, Antoniadou A, Giamarellou H, Petrikkos GL, et al. The association between colonization with carbapenemase-producing enterobacteriaceae and overall ICU mortality: an observational cohort study. Crit Care Med. 2015;43(6):1170-7.

37. Falcone M, Russo A, lacovelli A, Restuccia G, Ceccarelli G, Giordano A, et al. Predictors of outcome in ICU patients with septic shock caused by Klebsiella pneumoniae carbapenemase-producing K. pneumoniae. Clin Microbiol Infect 2016;22(5):444-50.

38. Hussein K, Raz-Pasteur A, Finkelstein R, Neuberger A, Shachor-Meyouhas Y, Oren I, et al. Impact of carbapenem resistance on the outcome of patients hospital-acquired bacteraemia caused by Klebsiella pneumoniae. J Hosp Infect. 2013;83(4):307-13

39. Kuntaman K, Shigemura K, Osawa K, Kitagawa K, Sato K, Yamada N, et al. Occurrence and characterization of carbapenem-resistant gram-negative bacilli: a collaborative study of antibiotic-resistant bacteria between Indonesia and Japan. Int J Urol. 2018;25(11):966-72.

40. Liu H, Fei CN, Zhang Y, Liu GW, Liu J, Dong J. Presence, distribution and molecular epidemiology of multi-drug-resistant gram-negative bacilli from medical personnel of intensive care units in Tianjin, China, 2007-2015. J Hosp Infect. 2017;96(2):101-10.

41. Wei WJ, Yang HF, Ye Y, Li JB. New Delhi Metallo-beta-lactamase-mediated Carbapenem resistance: origin, diagnosis, treatment and public health concern. Chin Med J. 2015;128(14):1969-76.

42. Bouguenoun W, Bakour S, Bentorki AA, Al Bayssari C, Merad T, Rolain JM Molecular epidemiology of environmental and clinical carbapenemaseproducing gram-negative bacilli from hospitals in Guelma, Algeria: multiple genetic lineages and first report of OXA-48 in Enterobacter cloacae. J Glob Antimicrob Resist. 2016;7:135-40.

43. Yagoubat M, Ould El-Hadj-Khelil A, Malki A, Bakour S, Touati A, Rolain JM. Genetic characterisation of carbapenem-resistant gram-negative bacteria isolated from the university hospital Mohamed Boudiaf in Ouargla, southern Algeria. J Glob Antimicrob Resist. 2016;8:55-9.

44. Zheng R, Zhang Q, Guo Y, Feng Y, Liu L, Zhang A, et al. Outbreak of plasmid-mediated NDM-1-producing Klebsiella pneumoniae ST105 among neonatal patients in Yunnan, China. Ann Clin Microbiol Antimicrob. 2016;15:10.
45. Wang X, Xu X, Li Z, Chen H, Wang Q, Yang P, et al. An outbreak of a nosocomial NDM-1-producing Klebsiella pneumoniae ST147 at a teaching hospital in mainland China. Microb Drug Resist. 2014;20(2):144-9.

46. Wu W, Feng Y, Tang G, Qiao F, McNally A, Zong Z. NDM Metallo-betaLactamases and Their Bacterial Producers in Health Care Settings. Clin Microbiol Rev. 2019;32(2):e00115-8.

\section{Publisher's Note}

Springer Nature remains neutral with regard to jurisdictional claims in published maps and institutional affiliations.
Ready to submit your research? Choose BMC and benefit from:

- fast, convenient online submission

- thorough peer review by experienced researchers in your field

- rapid publication on acceptance

- support for research data, including large and complex data types

- gold Open Access which fosters wider collaboration and increased citations

- maximum visibility for your research: over $100 \mathrm{M}$ website views per year

At BMC, research is always in progress.

Learn more biomedcentral.com/submissions 